\title{
Fisher Zeros and Correlation Decay in the Ising Model
}

\author{
Jingcheng Liu ${ }^{1}$ \\ Computer Science Division, UC Berkeley, USA \\ liuexp@berkeley.edu
}

\author{
Alistair Sinclair ${ }^{2}$ \\ Computer Science Division, UC Berkeley, USA \\ sinclair@cs.berkeley.edu
}

\section{Piyush Srivastava ${ }^{3}$}

Tata Institute of Fundamental Research, Mumbai, India

piyush.srivastava@tifr.res.in

\begin{abstract}
The Ising model originated in statistical physics as a means of studying phase transitions in magnets, and has been the object of intensive study for almost a century. Combinatorially, it can be viewed as a natural distribution over cuts in a graph, and it has also been widely studied in computer science, especially in the context of approximate counting and sampling. In this paper, we study the complex zeros of the partition function of the Ising model, viewed as a polynomial in the "interaction parameter"; these are known as Fisher zeros in light of their introduction by Fisher in 1965. While the zeros of the partition function as a polynomial in the "field" parameter have been extensively studied since the classical work of Lee and Yang, comparatively little is known about Fisher zeros. Our main result shows that the zero-field Ising model has no Fisher zeros in a complex neighborhood of the entire region of parameters where the model exhibits correlation decay. In addition to shedding light on Fisher zeros themselves, this result also establishes a formal connection between two distinct notions of phase transition for the Ising model: the absence of complex zeros (analyticity of the free energy, or the logarithm of the partition function) and decay of correlations with distance. We also discuss the consequences of our result for efficient deterministic approximation of the partition function. Our proof relies heavily on algorithmic techniques, notably Weitz's self-avoiding walk tree, and as such belongs to a growing body of work that uses algorithmic methods to resolve classical questions in statistical physics.
\end{abstract}

2012 ACM Subject Classification Mathematics of computing $\rightarrow$ Discrete mathematics

Keywords and phrases Ising model, zeros of polynomials, partition functions, approximate counting, phase transitions

Digital Object Identifier 10.4230/LIPIcs.ITCS.2019.55

Related Version Full version available at [15], https://arxiv.org/abs/1807.06577.

\footnotetext{
1 Supported by US NSF grant CCF-1815328.

2 Supported by US NSF grant CCF-1815328.

3 Supported by a Ramanujan Fellowship of the Indian Department of Science and Technology.

cc) (i) Jingcheng Liu, Alistair Sinclair, and Piyush Srivastava;

(c) licensed under Creative Commons License CC-BY
10th Innovations in Theoretical Computer Science (ITCS 2019)

Editor: Avrim Blum; Article No. 55; pp. 55:1-55:8

Leibniz International Proceedings in Informatics 


\section{Introduction}

In combinatorial terms, the Ising model is a probability distribution over the cuts of a graph. Given a graph $G=(V, E)$, the configurations of the model are assignments $\sigma$ of "+" or "-" spins to the vertices of $G$; $\sigma$ corresponds to the cut between spin-"+" and spin-"-" vertices. The model assigns to configuration $\sigma$ the weight $w_{G, \beta}(\sigma)=\beta^{|\{e=(u, v) \in E: \sigma(u) \neq \sigma(v)\}|}$, where $\beta>0$ is a parameter. The associated probability distribution, known as the Gibbs measure, is then defined by assigning probability $\mu_{G, \beta}(\sigma):=\frac{1}{Z_{G}(\beta)} w_{G, \beta}(\sigma)$ to each configuration $\sigma$. The normalizing factor here is the partition function, defined as

$$
Z_{G}(\beta):=\sum_{\sigma: V \rightarrow\{+,-\}} w_{G, \beta}(\sigma)=\sum_{k=0}^{|E|} \gamma_{k} \beta^{k}
$$

where $\gamma_{k}$ is the number of $k$-edge cuts in $G$. Note that $Z_{G}(\beta)$ is a polynomial in $\beta$ with positive coefficients. We also sometimes consider graphs in which certain vertices are pinned to "+" or "-" spins. For such a graph, we restrict the sum in the definition of $Z_{G}$ to those configurations $\sigma$ in which these vertices have the spin determined by their pinning.

The origins of the Ising model lie in the qualitative modeling of phase transitions in magnets [11]; indeed, it was the first model among the wide class of spin systems to be studied extensively in statistical physics. The parameter $\beta$ above is a proxy for the "temperature" or "interaction strength", while the graph is a proxy for the physical structure of the magnet. In this parameterization, $\beta>1$ corresponds to so-called anti-ferromagnetic interactions (where neighbors prefer to have different spins), $\beta<1$ to ferromagnetic interactions (where neighbors prefer to have the same spins), and $\beta=1$ to infinite temperature (where the neighbors behave independently of each other). We will restrict our attention throughout to graphs of fixed (but arbitrary) maximum degree $\Delta$.

Historically, there have been two distinct (though closely related) mechanisms for defining and understanding phase transitions in statisical physics. The first is decay of long-range correlations in the Gibbs measure, which is familiar in theoretical computer science due to its extensive use in approximation algorithms and the analysis of spin systems and graphical models. The second, which is more classical and less familiar in computer science, is analyticity of the "free energy" $\log Z$ (where $Z$ is the partition function). This second notion connects naturally to stability theory of polynomials, and in particular to the study of the location of complex roots of the partition function $Z$, even when only real values of the parameters make physical sense in the model. The seminal work of Lee and Yang $[12,28]$ was one of the first, and certainly the best known, to use this notion. We note in passing that stability theory has seen a surge of recent interest in theoretical computer science, in contexts ranging from approximation algorithms to the construction of Ramanujan graphs (see, e.g., $[17,18,1,2,26])$.

We now briefly describe the connection between the analyticity of the free energy and the location of complex zeros of the partition function. The first ingredient is that natural observables of the model (e.g., the magnetization) can be written as derivatives of the free energy with respect to an appropriate parameter of the model. Thus, analyticity of the free energy for a given range $S$ of parameters implies that all such observables vary continuously (and have continuous derivatives) when the parameter value lies in $S$, which in turn implies that there is no phase transition in $S$. However, it is not hard to see that for any finite graph, the free energy is always analytic as a function of $\beta$ when $\beta$ lies on the positive real axis, suggesting a complete absence of phase transitions. Indeed, it turns out (see, e.g., [23, Chapter 1]) that in order to see phase transitions one has to consider infinite graphs. 
For concreteness, we consider the case of the Ising model on the infinite 2-dimensional integer lattice $\mathbb{Z}^{2}[28]$. In order to define the free energy for such an infinite graph, one takes the limit of the free energies of a suitable increasing sequence of finite subgraphs (e.g., increasing rectangles in $\mathbb{Z}^{2}$ ), after scaling them by their size. Lee and Yang [28] showed that, for infinite graphs of sub-exponential growth (including $\mathbb{Z}^{2}$ ), the free energy obtained via this prescription is well defined and analytic for a range of parameters $S$ provided that the partition functions of the finite graphs used in the limit definition, viewed as polynomials in the parameter, are zero-free in a complex neighborhood of $S$. Thus, proving zero-freeness of partition functions of such a sequence of finite graphs in a fixed (i.e., not depending upon the finite graphs in question) complex neighborhood of $S$ implies the absence of phase transitions in $S$.

Algorithms, phase transitions, and roots of polynomials. While the algorithmic consequences of phase transitions defined in terms of decay of correlations have been well studied, first in the context of Markov Chain Monte Carlo algorithms (Glauber dynamics) and more recently in determinstic algorithms that directly exploit correlation decay, algorithmic use of the information on complex roots of the partition function originated only recently in the work of Barvinok (see [3] for a survey). This has led to increased interest in understanding the relationship between the above two notions of phase transitions. Such connections have been the focus of some recent work on the independent set (or "hard core lattice gas") model; notably, connections similar to the ones in this paper have been explored for that model by Peters and Regts [20], while related ideas are harnessed in early work of Shearer [22], as later elucidated by Scott and Sokal [21] and further elaborated by Harvey et al. [10], to shed light on the Lovász Local Lemma.

The motivation for our work here is to take a step towards achieving a fuller understanding of these connections. Specifically, we study the zeros of the Ising partition function (at zero field), viewed as a polynomial in the interaction parameter. While the study of zeros in terms of the fugacity (or field) parameter was famously pioneered by Lee and Yang [12], and has given rise to a well developed theory, very little is known about the zeros in terms of the interaction parameter, which were first studied in the classical 1965 paper of Fisher [8] and are thus known as "Fisher zeros".

Our main result is that the Ising model has no Fisher zeros in a region of the complex plane that contains the entire interval $B$ on the positive real line where correlation decay holds. Our analysis crucially exploits the correlation decay property in order to understand the Fisher zeros. Thus, in the particular case of the zero field Ising model, we are able to establish a tight connection between correlation decay and the absence of zeros. Another potentially interesting aspect of this result is the use of algorithmic techniques associated with correlation decay (notably, Weitz's algorithm [27]) to understand a classical concept in statistical physics.

We now proceed to formally describe our results. First we identify the range of the parameter $\beta$ for which the Ising model is, in a certain sense, well-behaved on graphs of bounded degree $\Delta$.

- Definition 1 (Correlation decay region). Given $\Delta>0$, the correlation decay region $B=B_{\Delta}$ for $\beta$ is the interval $\left(\frac{\Delta-2}{\Delta}, \frac{\Delta}{\Delta-2}\right)$.

The correlation decay region is very well studied in both physical and algorithmic contexts, and comes from a consideration of the behavior of the Gibbs measure on trees. In particular, it corresponds to those $\beta$ for which there is exponential decay of correlations in the Gibbs measure on any finite subtree of the infinite $\Delta$-regular tree - a fact which has been used to 
give a deterministic algorithm for approximating the partition function of the Ising model for such $\beta[27,29]$. On the other hand, Sly and Sun [25] have shown that for $\beta>\frac{\Delta}{\Delta-2}$, this approximation problem is NP-hard under randomized reductions. In statistical physics, the correlation decay region describes those $\beta$ for which the definition of the Gibbs measure given by eq. (1) for finite graphs can be extended in a unique way to a Gibbs measure on the infinite $\Delta$-regular tree [9]; for this reason, the correlation decay region is also referred to as the uniqueness region.

As advertised earlier, our goal is to prove the existence of a region of the complex plane, containing $B$, which contains no Fisher zeros. We state this now as our main theorem.

- Theorem 2. Fix any $\Delta>0$. For any real $\beta \in B:=\left(\frac{\Delta-2}{\Delta}, \frac{\Delta}{\Delta-2}\right)$, there exists a $\delta>0$ such that for all $\beta^{\prime} \in \mathbb{C}$ with $\left|\beta^{\prime}-\beta\right|<\delta$, the Ising partition function $Z_{G}\left(\beta^{\prime}\right) \neq 0$ for all graphs $G$ of maximum degree $\Delta$. Moreover the same holds even if $G$ contains an arbitrary number of vertices pinned to + or - spins.

- Remarks.

(1) It is worth noting that the choice of $\delta$ does not depend on the size of the graph, only on $\Delta$ and $\beta$. In particular, given any $\delta_{1}>0$, one can choose $\delta>0$ such that, for all $\beta^{\prime}$ in a complex neighborhood of radius $\delta$ around the closed interval $\left[\frac{\Delta-2}{\Delta}+\delta_{1}, \frac{\Delta}{\Delta-2}-\delta_{1}\right]$, $Z_{G}\left(\beta^{\prime}\right)$ is non-zero for all graphs of degree at most $\Delta$.

(2) For the case of the Ising model, the above theorem establishes a connection between the two notions of phase transition discussed above. Namely, for the zero-field Ising model, it shows that decay of correlations on the $\Delta$-regular tree also implies the absence of Fisher zeros for finite graphs of degree at most $\Delta$, and hence the analyticity of the free energy for appropriate infinite graphs (i.e., those of maximum degree at most $\Delta$ and of subexponential growth, such as regular lattices).

Discussion. To the best of our knowledge, the previous best general result on the Fisher zeros of the Ising model appears in the work of Barvinok and Soberón [6], who showed that $Z_{G}(\beta)$ is non-zero if $|\beta-1|<c / \Delta$, where $\Delta$ is the maximum degree of $G$, and $c$ can be chosen to be 0.34 (and as large as 0.45 if $\Delta$ is large enough). While this result provides a disk around 1 in which there are no Fisher zeros, it cannot guarantee the absence of Fisher zeros in a neighborhood of the correlation decay region $B$ (which would require at least that $\left.c \geq 2-o_{\Delta}(1)\right)$. Our Theorem 2 therefore strengthens this result to a neighborhood of the entire correlation decay region $B{ }^{4}$

Our main theorem on Fisher zeros can also be combined with the techniques of Barvinok [3] and Patel and Regts [19] to give a new deterministic polynomial time approximation algorithm for the partition function of the ferromagnetic Ising model with zero field on graphs of degree at most $\Delta$ when $\beta \in\left(\frac{\Delta-2}{\Delta}, \frac{\Delta}{\Delta-2}\right)$. In particular, combining Theorem 2 with Lemmas 2.2.1 and 2.2.3 of [3] (see also the discussion at the bottom of page 27 therein) and the proof of Theorem 6.1 of [19], we obtain the following corollary:

- Corollary 3. Fix a positive integer $\Delta$ and $\delta>0$. There exist positive constants $\delta_{1}>0$ and $c$ such that for any complex $\beta$ with $\Re(\beta) \in\left[\frac{\Delta-2}{\Delta}+\delta, \frac{\Delta}{\Delta-2}-\delta\right]$ and $|\Im(\beta)| \leq \delta_{1}$, the following is true. There exists an algorithm which, on input a graph $G$ of degree at most $\Delta$ on $n$ vertices, and an accuracy parameter $\varepsilon>0$, runs in time $O(n / \varepsilon)^{c}$ and outputs $\hat{Z}$ satisfying $\left|\hat{Z}-Z_{G}(\beta)\right| \leq \varepsilon\left|Z_{G}(\beta)\right|$.

4 Technically the results are incomparable in the sense that, while our results cover a much larger portion of the real line than that in [6], the diameter of the disk centered around 1 in the region of [6] may be larger than the radius guaranteed by our result. 
For real $\beta$ in the same range, a deterministic algorithm with the above properties, based on correlation decay, was already analyzed in [29]. However, our extension to complex values of the parameter is also interesting in light of the fact that algorithms for approximating the Ising partition function at complex values of the parameters have recently been studied in the context of classical simulation of restricted models of quantum computation [16].

Finally, we emphasize that in contrast to most other recent applications of Barvinok's method (e.g., [19, 5, 6, 4, 14]), where the required results on the location of the roots of the associated partition function are derived without reference to correlation decay, the algorithmic version of correlation decay is crucial to our proof. Indeed, implicit in our proof is an analysis of Weitz's celebrated correlation decay algorithm [27] (proposed originally for the independent set (or "hard core") model, and analyzed by Zhang, Liang and Bai [29] for the Ising model in the case of real positive $\beta \in B$ ) for the Ising model with complex $\beta^{\prime}$ close to $\beta \in B$. Thus, as mentioned earlier, our work shows that Weitz's algorithm can be viewed as a bridge between the "decay of correlations" and "analyticity of free energy" views of phase transitions. We note also that our work is close in spirit to recent work of Peters and Regts [20] (see also [7]), who employ correlation decay in the hard core model to prove stability results for the hard core partition function.

\section{Outline of proof}

We fix $\Delta$ to be the maximum degree throughout, and let $d=\Delta-1$. Let $G$ be any graph of maximum degree $\Delta$. Our starting point is a recursive criterion that guarantees that the partition function $Z_{G}(\beta)$ has no zeros. For any non-isolated vertex $v$ of $G$, let $Z_{G, v}^{+}(\beta)$ (respectively, $Z_{G, v}^{-}(\beta)$ ) be the contribution to $Z_{G}(\beta)$ from configurations with $\sigma(v)=+$ (respectively, with $\sigma(v)=-$ ), so that $Z_{G}(\beta)=Z_{G, v}^{+}(\beta)+Z_{G, v}^{-}(\beta)$. Define also the ratio $R_{G, v}(\beta):=\frac{Z_{G, v}^{+}(\beta)}{Z_{G, v}^{-}(\beta)}$. Now note that $Z_{G, v}^{+}(\beta)$ and $Z_{G, v}^{-}(\beta)$ can be seen as Ising partition functions defined on the same graph $G$ with the vertex $v$ pinned to the appropriate spin; i.e., they are partition functions defined on a graph with one less unpinned vertex. Thus we may assume recursively that neither $Z_{G, v}^{+}(\beta)$ nor $Z_{G, v}^{-}(\beta)$ vanishes. Under this assumption, the condition $Z_{G}(\beta) \neq 0$ is equivalent to $R_{G, v}(\beta) \neq-1$.

Our next ingredient is a formal recurrence, due to Weitz [27], for computing ratios such as $R_{G, v}(\beta)$ in two-state spin systems. This recurrence is based on the so-called "tree of self-avoiding walks" (or "SAW tree") in $G$, rooted at $v$, with appropriate boundary conditions (i.e., initial inputs, or fixed values at the leaves of the tree). Weitz's recurrence has been used in the development of several approximate counting algorithms based on decay of correlations (see, e.g., $[27,29,13,24])$. We now state a precise version of Weitz's result that is tailored to our application.

- Lemma 4. Let $G$ be a graph of maximum degree $\Delta=d+1$, with some vertices possibly pinned to spins "+" or "-". Given $\beta \in \mathbb{C}$, define $h_{\beta}(x):=\frac{\beta+x}{\beta x+1}$. For integers $k \geq 0$ and $s$, define the maps

$$
F_{\beta, k, s}(\boldsymbol{x}):=\beta^{s} \prod_{i=1}^{k} h_{\beta}\left(x_{i}\right) .
$$

Then, the ratio $R_{G, v}(\beta)$ can be obtained by iteratively applying a sequence of multivariate maps of the form $F_{\beta, k, s}(\boldsymbol{x})$ such that, in all but the final application, one has $1 \leq k+|s| \leq d$, while for the final application one has $1 \leq k+|s| \leq \Delta$, and any initial input to these maps is $x_{i}=1$. 
For completeness we sketch a proof of Lemma 4 at the end of this section.

Returning now to the condition $R_{G, v}(\beta) \neq-1$ derived above, we see from Lemma 4 that a sufficient condition for the absence of zeros of $Z_{G}(\beta)$ is the existence of a subset $D \subseteq \mathbb{C}$ such that $1 \in D,-1 \notin D$, and $D$ is closed under the recurrence $F_{\beta, k, s}$ (in the sense that $F_{\beta, k, s}$ maps $D^{k}$ into $\left.D\right)$. These properties guarantee that the recurrence, with initial inputs 1 at the leaves, can never yield the value -1 , and hence that $R_{G, v}(\beta) \neq-1$, so $Z_{G}(\beta) \neq 0$. The main technical content of this paper is to prove, under the conditions on $\beta$ stated in Theorem 2, the existence of such a set $D$, a result which we formally state as follows.

- Theorem 5. Fix a degree $\Delta=d+1$. For any $\beta \in\left(\frac{\Delta-2}{\Delta}, \frac{\Delta}{\Delta-2}\right)$, there exists $\delta_{\beta}>0$ such that, for any $\beta^{\prime} \in \mathbb{C}$ with $\left|\beta^{\prime}-\beta\right| \leq \delta_{\beta}$, there exists a set $D \subseteq \mathbb{C}$ with $1 \in D,-1 \notin D$, and

(a) $F_{\beta^{\prime}, k, s}\left(D^{k}\right) \subseteq D$ for integers $k \geq 0$ and $s$ such that $1 \leq k+|s| \leq d$;

(b) $-1 \notin F_{\beta^{\prime}, k, s}\left(D^{k}\right)$ for integers $k \geq 0$ and $s$ such that $1 \leq k+|s| \leq \Delta$.

At the end of this section, we spell out the details of how to combine Lemma 4 and Theorem 5 into a proof of our main result, Theorem 2 .

The main technical task of the paper is to prove Theorem 5 . We briefly sketch our approach in this extended abstract; the details can be found in the full version [15]. The first step is to simplify the problem by working with a univariate version of the recurrence $F_{\beta, k, s}$ defined in Lemma 4. The univariate version is defined as $f_{\beta, k, s}(x):=\beta^{s} h_{\beta}(x)^{k}$, and we can show that it satisfies $F_{\beta}\left(D^{k}\right)=f_{\beta}(D)$ for any set $D$ such that $C:=\log \left(h_{\beta}(D)\right)$ is convex in the complex plane. (Henceforth we will drop the subscripts $k, s$ for simplicity.) This means that the set $D$ we seek in Theorem 5 should be the image of a convex set $C$ under the map $\log \circ h_{\beta}$.

Next, to enable us to exploit the fact that $\beta$ is in the correlation decay interval $B=$ $\left(\frac{\Delta-2}{\Delta}, \frac{\Delta}{\Delta-2}\right)$, we further modify the univariate recurrence to $f_{\beta}^{\varphi}:=\varphi \circ f_{\beta} \circ \varphi^{-1}$, where $\varphi(x):=\log x$. This is an example of the use of a so-called "potential" function $\varphi$ in order to smooth a recurrence, as has been useful in several correlation decay arguments. The key point here is that, when $\beta \in B, f_{\beta}^{\varphi}$ (unlike $f_{\beta}$ itself) is actually a uniform contraction on an appropriate domain in $\mathbb{C}$; hence we can conclude that $f_{\beta}^{\varphi}(S) \subseteq S$ for "nice" sets $S$ (i.e., $S$ that are convex and symmetric around the origin). Since the condition $f_{\beta}(D) \subseteq D$ is equivalent to $f_{\beta}^{\varphi}(\log D) \subseteq \log D$, this imposes the further constraint that $\log D$ be a "nice" set.

Putting together the constraints in the previous two paragraphs, we need to construct a suitable convex set $C$ whose image $\log \left(h_{\beta}^{-1}(\exp (C))\right)$ is nice; our set $D$ in Theorem 5 will then be defined as $h_{\beta}^{-1}(\exp (C))$ (and this set must include 1 and exclude -1 ). This turns out to be hard to achieve directly due to the complexity of the map $p:=\log \circ h_{\beta}^{-1} \circ \exp$. However, we are able to show that one can instead work with a (non-analytic) approximation of $p$ under which the image of a natural convex $C$ becomes a nice (in fact, rectangular) set. Moreover, this holds even for complex $\beta$ that are sufficiently close to the region $B$. This fact then allows us to push through the analysis and arrive at a proof of Theorem 5 .

We conclude this overview with the proofs of Lemma 4 and Theorem 2 promised earlier. The proof of our main technical result, Theorem 5 , can be found in the full version [15].

Proof of Lemma 4 (Sketch). This description is exactly the same as the version of Weitz's result used for the Ising model in, e.g., [24] and [29], except that there, only the maps $F_{\beta, k, 0}$ for $1 \leq k \leq d$ (with at most one final application with $k=\Delta$ ) are used, and the initial values come from the set $\{0, \infty\}$; these initial values are the values of the ratio for single leaf vertices in the SAW tree pinned to - and + respectively, and the maps $F_{\beta, k, 0}$ describe how to combine the ratios from $k$ subtrees. The version in the lemma follows by 
noticing that $h_{\beta}(1)=1, h_{\beta}(0)=\beta$ and $h_{\beta}(\infty)=1 / \beta$, so that $F_{\beta, k, 0}$ applied to a vector $\boldsymbol{x}$ with $k$ coordinates, $s_{1}$ of which are set to 0 and $s_{2}$ to $\infty$, produces the same output as $F_{\beta, k-\left|s_{1}-s_{2}\right|, s_{1}-s_{2}}$ applied to the vector $\boldsymbol{x}^{\prime}$ of $k-\left|s_{1}-s_{2}\right|$ coordinates obtained from $\boldsymbol{x}$ by removing the 0 and $\infty$ entries, and then appending $s_{1}+s_{2}-\left|s_{1}-s_{2}\right|$ entries which are 1 .

Proof of Theorem 2. As indicated earlier, the induction is on the number of unpinned vertices, $n$, of $G$. For the base case $n=0, Z_{G}(\beta)=\beta^{k}$, where $k$ is the number of pairs of adjacent vertices in $G$ that are pinned to different spins. Therefore, $Z_{G}(\beta) \neq 0$ unless $\beta=0$. Next suppose that for some positive integer $t$, it holds that for every $\beta \in B$, there exists a $\delta>0$ such that for all $\beta^{\prime} \in \mathbb{C}$ with $\left|\beta^{\prime}-\beta\right|<\delta, Z_{G}\left(\beta^{\prime}\right) \neq 0$ for all graphs $G$ of maximum degree $\Delta$ with at most $t$ unpinned vertices. Now, let $G^{\prime}$ be any graph of the same maximum degree with $t+1$ unpinned vertices. Fix any non-isolated vertex $v$ in $G^{\prime}$, and let $Z_{G^{\prime}, v}^{+}\left(\beta^{\prime}\right), Z_{G^{\prime}, v}^{-}\left(\beta^{\prime}\right)$ be the contributions to the partition function from configurations with $\sigma(v)=+, \sigma(v)=-$, respectively. By the induction hypothesis, we know that $Z_{G^{\prime}, v}^{+}\left(\beta^{\prime}\right) \neq 0, Z_{G^{\prime}, v}^{-}\left(\beta^{\prime}\right) \neq 0$ as they are exactly the Ising partition function defined on the same graph $G^{\prime}$ with the vertex $v$ pinned (thus reducing the number of unpinned vertices to $t$ ). Further, Lemma 4 implies that $R_{G^{\prime}, v}\left(\beta^{\prime}\right)=\frac{Z_{G^{\prime}, v}^{+}{ }^{\left(\beta^{\prime}\right)}}{Z_{G^{\prime}, v}^{-}\left(\beta^{\prime}\right)}$ can be computed by iteratively applying a sequence of maps of the form $F_{\beta^{\prime}, k, s}$ for $1 \leq k+|s| \leq d$, followed by at most one application where $k+|s|=\Delta$, starting with initial values of 1 . Part (a) of Theorem 5 then implies that the outputs of all except possibly the final application remain in the set $D$ defined in that theorem, and part (b) of the theorem implies that the final output, which is equal to $R_{G^{\prime}, v}(\beta)$ by Lemma 4 , is not -1 . Since $Z_{G^{\prime}, v}^{+}\left(\beta^{\prime}\right)$ and $Z_{G^{\prime}, v}^{-}\left(\beta^{\prime}\right)$ are non-zero, this implies that $Z_{G^{\prime}}\left(\beta^{\prime}\right) \neq 0$, completing the induction.

\section{References}

1 Nima Anari and Shayan Oveis Gharan. The Kadison-Singer problem for strongly Rayleigh measures and applications to Asymmetric TSP. In Proc. 56th IEEE Symp. Found. Comp. Sci. (FOCS), October 2015.

2 Nima Anari and Shayan Oveis Gharan. A Generalization of Permanent Inequalities and Applications in Counting and Optimization. In Proc. 49th ACM Symp. Theory Comput. (STOC), pages 384-396. ACM, June 2017. arXiv:1702.02937.

3 A. Barvinok. Combinatorics and Complexity of Partition Functions. Algorithms and Combinatorics. Springer, 2017.

4 Alexander Barvinok. Computing the permanent of (some) complex matrices. Found. Comput. Math., 16(2):329-342, 2015. doi:10.1007/s10208-014-9243-7.

5 Alexander Barvinok and Pablo Soberón. Computing the partition function for graph homomorphisms with multiplicities. J. Combin. Theory, Ser. A, 137:1-26, 2016.

6 Alexander Barvinok and Pablo Soberón. Computing the partition function for graph homomorphisms. Combinatorica, 37(4):633-650, August 2017.

7 Ferenc Bencs and Péter Csikvári. Note on the zero-free region of the hard-core model, July 2018. arXiv:1807.08963.

8 M. E. Fisher. The nature of critical points. In W. E. Brittin, editor, Lecture notes in Theoretical Physics, volume 7c, pages 1-159. University of Colorado Press, 1965.

9 Hans-Otto Georgii. Gibbs Measures and Phase Transitions. De Gruyter Studies in Mathematics. Walter de Gruyter Inc., October 1988.

10 Nicholas J. A. Harvey, Piyush Srivastava, and Jan Vondrák. Computing the independence polynomial: from the tree threshold down to the roots. In Proc. 29th ACM-SIAM Symp. Discrete Algorithms (SODA), pages 1557-1576, 2018. arXiv:1608.02282. 
11 E. Ising. Beitrag zur Theorie des Ferromagnetismus. Z. Phys., 31:253-258, February 1925. doi: $10.1007 /$ BF02980577.

12 T. D. Lee and C. N. Yang. Statistical Theory of Equations of State and Phase Transitions. II. Lattice Gas and Ising Model. Phys. Rev., 87(3):410-419, 1952. doi:10.1103/PhysRev. 87.410 .

13 Liang Li, Pinyan Lu, and Yitong Yin. Correlation Decay up to Uniqueness in Spin Systems. In Proc. 24th ACM-SIAM Symp. Discrete Algorithms (SODA), pages 67-84, 2013.

14 Jingcheng Liu, Alistair Sinclair, and Piyush Srivastava. The Ising Partition Function: Zeros and Deterministic Approximation. In Proc. 58th Annual IEEE Symp. Found. Comp. Sci. (FOCS), pages 986-997, 2017. arXiv:1704.06493.

15 Jingcheng Liu, Alistair Sinclair, and Piyush Srivastava. Fisher zeros and correlation decay in the Ising model, 2018. arXiv:1807.06577.

16 Ryan L. Mann and Michael J. Bremner. Approximation algorithms for complex-valued Ising models on bounded degree graphs, June 2018. arXiv:1806.11282.

17 Adam Marcus, Daniel Spielman, and Nikhil Srivastava. Interlacing families I: Bipartite Ramanujan graphs of all degrees. Ann. Math., pages 307-325, July 2015. doi:10.4007/ annals.2015.182.1.7.

18 Adam W Marcus, Daniel A Spielman, and Nikhil Srivastava. Interlacing families II: Mixed characteristic polynomials and the Kadison-Singer problem. Ann. Math., 182:327-350, 2015.

19 Viresh Patel and Guus Regts. Deterministic polynomial-time approximation algorithms for partition functions and graph polynomials. SIAM J. Comput., 46(6):1893-1919, December 2017. arXiv:1607.01167.

20 Han Peters and Guus Regts. On a conjecture of Sokal concerning roots of the independence polynomial, January 2017. arXiv:1701.08049.

21 Alex Scott and Alan Sokal. The repulsive lattice gas, the independent-set polynomial, and the Lovász local lemma. J. Stat. Phys., 118(5-6):1151-1261, 2004.

22 James B. Shearer. On a problem of Spencer. Combinatorica, 5(3), 1985.

23 Barry Simon. The Statistical Mechanics of Lattice Gases, volume 1. Princeton University Press, 1993.

24 Alistair Sinclair, Piyush Srivastava, and Marc Thurley. Approximation Algorithms for Two-State Anti-Ferromagnetic Spin Systems on Bounded Degree Graphs. J. Stat. Phys., 155(4):666-686, 2014.

25 Allan Sly and Nike Sun. Counting in two-spin models on $d$-regular graphs. Ann. Probab., 42(6):2383-2416, November 2014. doi:10.1214/13-A0P888.

26 Damian Straszak and Nisheeth K. Vishnoi. Real Stable Polynomials and Matroids: Optimization and Counting. In Proc. 49th ACM Symp. Theory Comput. (STOC), pages 370-383. ACM, June 2017. arXiv:1611.04548.

27 Dror Weitz. Counting Independent Sets up to the Tree Threshold. In Proc. 38th ACM Symp. Theory Comput. (STOC), pages 140-149, 2006.

28 C. N. Yang and T. D. Lee. Statistical Theory of Equations of State and Phase Transitions. I. Theory of Condensation. Phys. Rev., 87(3):404-409, August 1952. doi:10.1103/PhysRev . 87.404 .

29 Jinshan Zhang, Heng Liang, and Fengshan Bai. Approximating partition functions of the two-state spin system. Inf. Process. Lett., 111(14):702-710, 2011. doi:10.1016/j.ipl. 2011.04 .012$. 